\title{
Anatomical Segmentectomy with a Hybrid VATS Approach in a Patient with Intralobar Pulmonary Sequestration after Severe Pneumonia: A Case Report
}

\author{
Soichi Shibuya ${ }^{1,2}$ Toru Nakamura $^{3} \quad$ Eiji Miyazaki $^{2}$ \\ ${ }^{1}$ Department of Pediatric General and Urogenital Surgery, Juntendo \\ University, Tokyo, Japan \\ 2 Department of Pediatric Surgery, Seirei Hamamatsu General \\ Hospital, Hamamatsu, Shizuoka, Japan \\ ${ }^{3}$ Department of Thoracic Surgery, Seirei Hamamatsu General \\ Hospital, Hamamatsu, Shizuoka, Japan \\ Eur J Pediatr Surg Rep 2017;5:e21-e25.
}

\begin{abstract}
Address for correspondence Soichi Shibuya, MD, PhD, Department of Pediatric General and Urogenital Surgery, Juntendo University, Tokyo 1300033, Japan (e-mail: soshibu@juntendo.ac.jp).
\end{abstract}

\begin{abstract}
Keywords

- pulmonary sequestration

- anatomical segmentectomy

- video-assisted thoracic surgery
\end{abstract}

\section{New Insights and the Importance for the Pediatric Surgeon}

Anatomical basal segmentectomy using a hybrid VATS approach was successfully performed in a child with intrapulmonary sequestration following a severe pneumonia. Hybrid VATS approach enables safety segmentectomy in children with magnified vision of thoracoscopy.

\section{Introduction}

Anatomical segmentectomy is an increasingly attractive alternative to lobectomy in selected adult patients. ${ }^{1,2}$ In children, however, the procedure is less common because of its technical complexity. Segmentectomy requires dividing the segmental bronchus and pulmonary vessels at the hilum. A history of pulmonary infection further increases the risk of complications. ${ }^{3}$

received

December 20, 2016

accepted after revision

April 24, 2017

\author{
DOI https://doi.org/ \\ 10.1055/s-0037-1603592. \\ ISSN 2194-7619.
}

Here, we present the case of a successful anatomical basal segmentectomy in a child with intralobar pulmonary sequestration (IPS) and recent pneumonia, using the invasive hybrid video-assisted thoracic surgery (VATS) approach. ${ }^{4}$

\section{Case Report}

A 6-year-old girl with a history of congenital cystic lung disease was referred to our hospital with persistent cough and fever

\footnotetext{
(c) 2017 Georg Thieme Verlag KG Stuttgart · New York
}

License terms

(c) (i) 


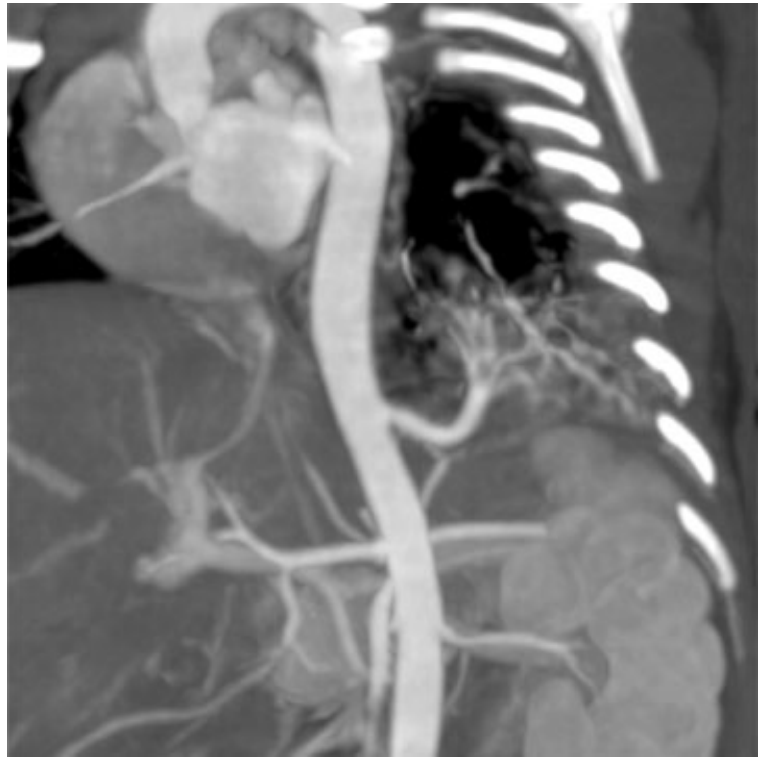

Fig. 1 Postnatal-enhanced computed tomography showing an anomalous arterial supply from the descending aorta to the left lower lobe.

refractory to oral antibiotic therapy. She was diagnosed antenatally with IPS in the left lung with anomalous arterial supply to the left basal segment ( - Fig. 1). She had no other symptoms prior to admission. Chest computed tomography (CT) showed diffuse consolidation of the left lower lobe. A diagnosis of bacterial pneumonia was made, and intravenous ceftriaxone was initiated. She was discharged after 5 days of treatment.

High-resolution CT showed a cystic lesion limited to the basal segment of the left lower lobe. The apical segment was hypertrophic, suggesting compensatory growth, with normal arterial perfusion, normal venous drainage, and a normal bronchial connection (-Fig. 2). A diagnosis of IPS was con- firmed, and elective basal segmentectomy with a hybrid VATS approach was planned.

Surgery was performed under general anesthesia with selected left bronchial occlusion, using a Fogarty catheter. The patient was placed in the right decubitus position. A $10-\mathrm{mm}$ thoracoscopic trocar was inserted through the ninth intercostal space in the left mid-axillary line. Thoracotomy was performed through the fifth intercostal space with a $5-\mathrm{cm}$ posterolateral incision. Inflammatory adhesions between the left lung and the chest wall were dissected under thoracoscopic vision. An aberrant artery running through the pulmonary ligament was detected. This was divided by silk thread ligation, clipping, and division using a bipolar system (the LigaSure vessel sealing system (Valleylab/Tyco Healthcare) (-Fig. 3a). The common basal vein was identified and divided posteriorly.

Following division of the major fissure, the basal arteries were cut individually (- Fig. 3b). Following confirmation of aeration in the apical segment, the basal bronchus was clamped and transected with an endoscopic linear stapler. The lung parenchyma was divided with the stapler on the inflation-deflation line between the affected basal segment and the healthy apical segment (-Fig.4). The specimen was retrieved through the thoracotomy without extension of the wound. The size of the specimen was $10 \times 9 \mathrm{~cm}$. The operative time was 217 minutes, and the estimated blood loss was $56 \mathrm{~mL}$.

Histopathological examination revealed a cystically dilated bronchus with inflammation of the surrounding parenchyma. The margin of the specimen did not include cystic lesion, confirming complete resection of the lesion. The wall of the aberrant artery was composed of elastic fibers. These findings were compatible with a diagnosis of IPS.

The patient recovered without complications and was discharged home on postoperative day 5 . A chest radiograph 4 months postprocedure demonstrated satisfactory expansion of residual lung (-Fig. 5). No respiratory impairment was reported throughout the 1-year follow-up period.
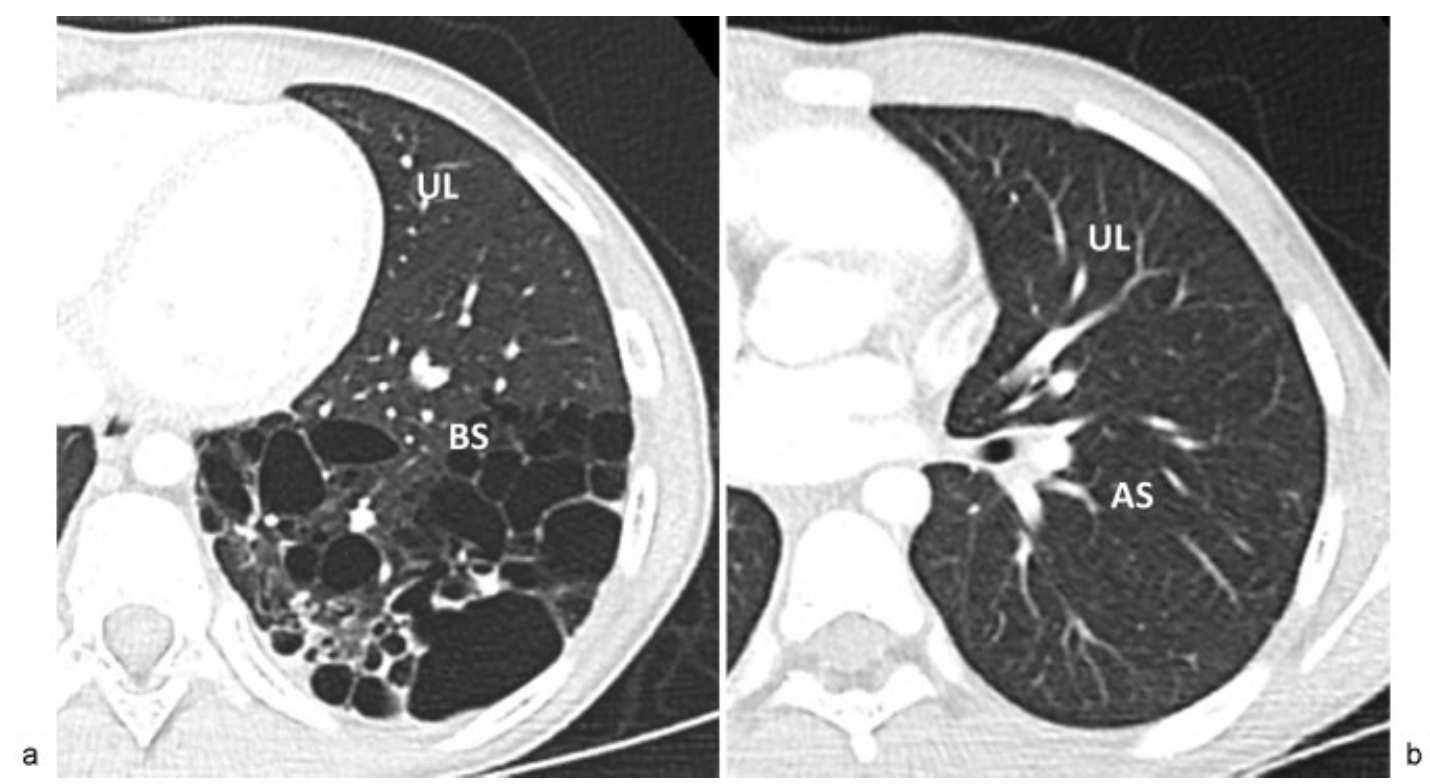

Fig. 2 Preoperative high-resolution computed tomography showing the affected BS with a normal AS. (a) UL, BS. (b) UL, AS. AS, apical segment; BS, basal segment; UL, upper lobe. 

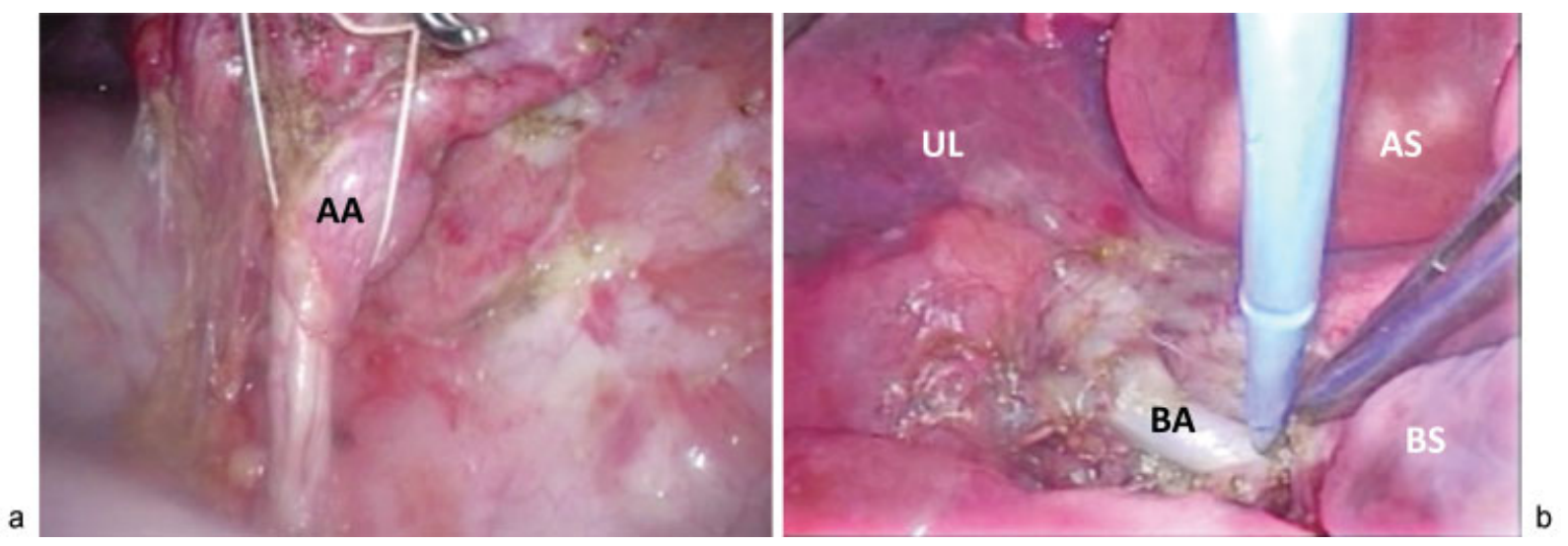

Fig. 3 Intraoperative view. (a) AA and (b) UL, AS, BS, BA. AA, aberrant artery; AS, apical segment; BA, basal artery; BS, basal segment; UL, upper lobe.

\section{Discussion}

Pulmonary sequestration is a congenital lung malformation defined as nonfunctional lung tissue without a bronchial connection to normal lung. ${ }^{5}$ In most cases, the abnormal lung tissue is supplied through an anomalous artery, usually arising from the descending aorta. This rare condition accounts for $\sim 1 \%$ of all congenital lung malformations. There are two subtypes, characterized according to their pleural covering: IPS, which is surrounded by the neighboring lung's pleura and extralobar pulmonary sequestration, which has its own pleura.

Definitive treatment of IPS is surgical resection to eliminate the possibility of recurrent respiratory infection, malignant transformation, and sudden hemoptysis caused by a ruptured anomalous artery. ${ }^{6}$

Lobectomy has been the most common surgical approach to IPS, as it is technically simple and affords reliable confirmation of complete resection. As recent advances in radiology have enabled surgeons to understand the detailed pulmonary anatomy preoperatively, lung-sparing surgeries such as segmentectomy and wedge resection are now considered viable options. $^{3}$

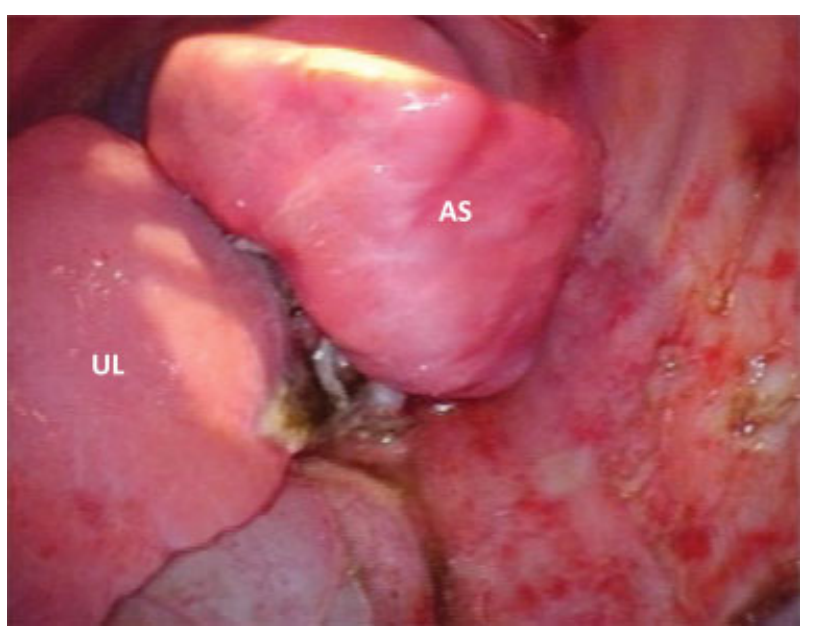

Fig. 4 Thoracoscopic view after basal segmentectomy showing preserved healthy apical segment. AS, apical segment; UL, upper lobe.
Anatomical segmentectomy is preferable to lobectomy as the former spares lung that has potential for compensatory growth following lung resection. One report suggested decreased exercise tolerance of children following lung resection. ${ }^{7}$ In older children, we do not expect a great deal compensatory lung growth following lung resection. Nevertheless, sparing healthy lung is valuable for preserving overall respiratory function, particularly if the healthy lung is hypertrophic, as in our case. ${ }^{8}$ The preserved lung can prevent thoracic deformity by expanding into the space created by resection. ${ }^{9}$ Finally, defined anatomical segmentectomy decreases the risk of residual lesion that often results from atypical resections. ${ }^{3,10}$

Another lung-sparing surgical approach is wedge resection, in which the affected lung is nonanatomically resected at the demarcation line. In wedge resection, sufficient space and margins are required to clamp the parenchyma with a surgical stapler. In the present case, the lesion was too large, and there was not adequate space to staple the parenchyma without risk of injuring hilar structures. The lesion occupied almost the entire basal segment, making it difficult to distinguish diseased tissue from normal parenchyma. We felt wedge resection risked leaving residual lesion. Therefore, we opted for anatomical segmentectomy.

Anatomical segmentectomy in this case presented several challenges. First, the patient had a history of pneumonia that might have caused severe adhesions in the thoracic cavity. Second, unlike other congenital lung malformations, resection of an IPS necessitates division of an anomalous artery. This requires meticulous manipulation deep in the thoracic cavity. To solve these problems, we employed a hybrid VATS approach with satisfactory results.

The efficacy of thoracoscopy in pediatric patients has been well documented over the past 20 years. ${ }^{11}$ Thoracoscopic surgery is a safe and effective procedure. The procedure decreases postoperative pain and length of hospital stay. ${ }^{12}$ However, the port-access feature limits the direction of view and mobility of surgical instruments. Therefore, thoracoscopic surgery tends to be avoided in complicated cases, such as newborns with comorbid congenital anomalies or respiratory infections. $^{13,14}$

Hybrid VATS is a variation of thoracoscopic surgery in which the procedure is performed using a combination of 


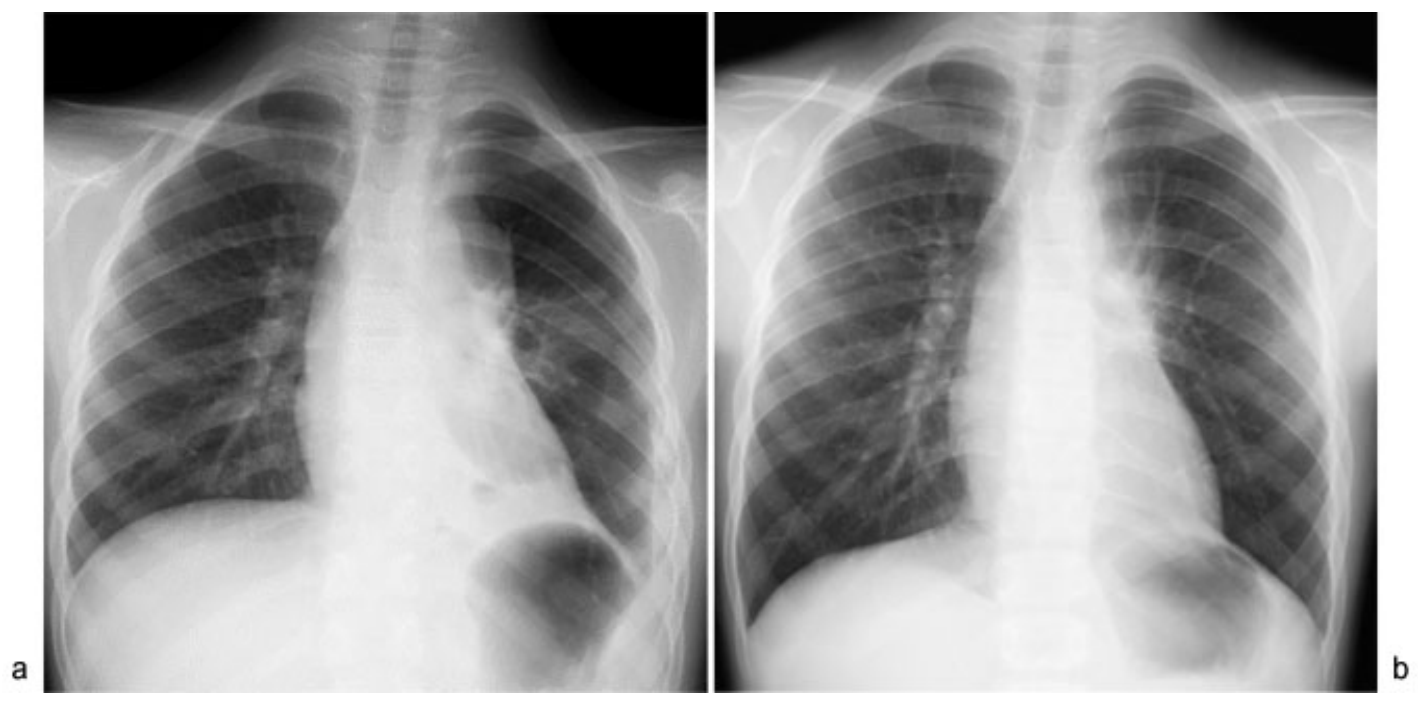

Fig. 5 Postoperative chest radiograph (a) 5 days after surgery and (b) 4 months after surgery.

direct view through a utility thoracotomy and magnified view via thoracoscopy.

Excision of an IPS entails the risk of tearing the aberrant artery and subsequent life-threatening hemorrhage. ${ }^{15}$ In our case, under magnification, the aberrant artery was easily distinguished from surrounding inflammatory tissues, and meticulous division was achieved despite the presence of a severe pneumonia.

There are reports of pediatric lung surgery employing a complete VATS approach without the use of a utility thoracotomy.${ }^{16}$ In our case, a complete VATS approach might have been unsuitable, as the lesion was too large to retrieve via a port site. We believe a 5-cm utility thoracotomy was necessary as well, as we required meticulous dissection and separation of tissues in a small and deep thoracic space.

There is some debate over timing of surgery for congenital pulmonary malformations, especially regarding risk in the presence of recent pneumonia. ${ }^{17}$ Rather than performing surgery when our patient was young and small, we adopted a "wait-and-see" approach, and delayed surgery until the infected sequestration became infected and was successfully treated. By this time, the patient was older and larger, both conditions being more favorable for resection. The patient's lung had healed from pneumonia well enough that the anatomical margin was easily distinguishable. This made lessinvasive segmentectomy more feasible. Although we found some adhesions between the lung and the parietal pleura, these could be dissected safely under thoracoscopic vision.

\section{Conclusion}

We safely performed an anatomical basal segmentectomy with magnified vision using a hybrid VATS approach in a child with IPS following a severe pneumonia. Our results suggest that the indications for hybrid VATS segmentectomy may be expanded further to include segmental lesions in children.

\section{References}

1 Nakamura K, Saji H, Nakajima R, et al. A phase III randomized trial of lobectomy versus limited resection for small-sized peripheral non-small cell lung cancer (JCOG0802/WJOG4607L). Jpn J Clin Oncol 2010;40(03):271-274

2 Schuchert MJ, Abbas G, Awais O, et al. Anatomic segmentectomy for the solitary pulmonary nodule and early-stage lung cancer. Ann Thorac Surg 2012;93(06):1780-1785 (discussion 1786-1787)

3 Fascetti-Leon F, Gobbi D, Pavia SV, et al. Sparing-lung surgery for the treatment of congenital lung malformations. J Pediatr Surg 2013;48(07):1476-1480

4 Okada M, Sakamoto T, Yuki T, Mimura T, Miyoshi K, Tsubota N. Hybrid surgical approach of video-assisted minithoracotomy for lung cancer: significance of direct visualization on quality of surgery. Chest 2005;128(04):2696-2701

5 Le Roux BT. Intralobar pulmonary sequestration. Thorax 1962; 17:77-84

6 Wang A, D’Amico TA, Berry MF. Surgical management of congenital pulmonary malformations after the first decade of life. Ann Thorac Surg 2014;97(06):1933-1938

7 Sritippayawan S, Treerojanapon S, Sanguanrungsirikul S, Deerojanawong J, Prapphal N. Pulmonary function and exercise capacity in children following lung resection surgery. Pediatr Surg Int 2012;28(12):1183-1188

8 Nakajima C, Kijimoto C, Yokoyama Y, et al. Longitudinal follow-up of pulmonary function after lobectomy in childhood - factors affecting lung growth. Pediatr Surg Int 1998;13(5-6):341-345

9 Kim HK, Choi YS, Kim K, et al. Treatment of congenital cystic adenomatoid malformation: should lobectomy always be performed? Ann Thorac Surg 2008;86(01):249-253

10 Johnson SM, Grace N, Edwards MJ, Woo R, Puapong D. Thoracoscopic segmentectomy for treatment of congenital lung malformations. J Pediatr Surg 2011;46(12):2265-2269

11 Rothenberg SS. Experience with thoracoscopic lobectomy in infants and children. J Pediatr Surg 2003;38(01):102-104

12 Kulaylat AN, Engbrecht BW, Hollenbeak CS, Safford SD, Cilley RE, Dillon PW. Comparing 30-day outcomes between thoracoscopic and open approaches for resection of pediatric congenital lung malformations: evidence from NSQIP. J Pediatr Surg 2015;50(10):1716-1721

13 Polites SF, Habermann EB, Zarroug AE, Thomsen KM, Potter DD. Thoracoscopic Vs open resection of congenital cystic lung diseaseutilization and outcomes in 1120 children in the United States. J Pediatr Surg 2016;51(07):1101-1105 
14 Sundararajan L, Parikh DH. Evolving experience with videoassisted thoracic surgery in congenital cystic lung lesions in a British pediatric center. J Pediatr Surg 2007;42(07):1243-1250

15 Peiry B, De Buys Roessingh A, Francini K, Alamo L, Reinberg O. Thoracoscopic segmentectomy: one vessel may hide a second one. J Pediatr Surg 2012;47(02):e11-e13
16 Inoue T, Oizumi H, Nakamura M, Sadahiro M. Port-access thoracoscopic anatomical segmentectomy for pediatric intralobar pulmonary sequestration. Thorac Cardiovasc Surg Rep 2014; 3(01):42-44

17 Peters RT, Burge DM, Marven SS. Congenital lung malformations: an ongoing controversy. Ann R Coll Surg Engl 2013;95(02):144-147 